\title{
ESTRATÉGIAS DE REDUÇÃO DE MODELOS MATEMÁTICOS APLICADAS AO PROCESSO DE PRODUÇÃO DE ETANOL EM BIORREATOR TUBULAR DE LEITO FIXO COM SEPARADORES EXTERNOS GÁS-LÍQUIDO
}

\author{
S. C. OLIVEIRA ${ }^{1}$, L. ZYMBERKNOPF ${ }^{2}$ e D. P. STREMEL ${ }^{3}$ \\ ${ }^{1}$ Faculdade de Ciências Farmacêuticas, UNESP - Univ Estadual Paulista, Departamento de \\ Bioprocessos e Biotecnologia. E-mail: samueloliveira@fcfar.unesp.br \\ ${ }^{2}$ SEE - Secretaria da Educação do Estado de São Paulo. E-mail: masterzymber@ gmail.com \\ ${ }^{3}$ Setor Palotina, UFPR - Universidade Federal do Paraná. E-mail: dile@ufpr.br
}

\begin{abstract}
RESUMO - Diferentes estratégias foram propostas para reduzir a complexidade de um modelo matemático de um processo contínuo de fermentação alcoólica em biorreator tubular de leito fixo. O modelo original incorpora, dentre outros fenômenos, transporte de massa pelo fluxo global, dispersão axial, transferência de massa interfásica e difusão intra-partícula. Simulações preliminares revelaram que os valores do fator de efetividade calculados para o biocatalisador foram próximos de um, indicando que os gradientes de concentração no interior da partícula podem ser negligenciados sem comprometer a capacidade preditiva do modelo. O comportamento hidrodinâmico do biorreator tubular foi aproximado por um modelo de N-CSTRs em série. Os balanços de massa em regime transiente foram resolvidos numericamente utilizando-se o método de integração de Runge-Kutta-Gill de quarta ordem. Os resultados obtidos permitiram verificar que as previsões do modelo reduzido com $\mathrm{N}=5$ coincidiram com as do modelo original e descreveram satisfatoriamente o comportamento observado das variáveis de processo.
\end{abstract}

\section{INTRODUÇÃO}

Existe um grande interesse na técnica de imobilização de células por encapsulamento em géis em razão das muitas vantagens que esta técnica pode oferecer. Com esta técnica pode-se atingir concentrações celulares mais elevadas do que aquelas atingidas em fermentadores convencionais com células livres e operar o reator a taxas de diluições maiores sem haver o risco de washout, aumentando assim a produtividade do processo.

Vários tipos de fermentadores contínuos para produção de etanol que operam com alta produtividade têm sido propostos na literatura, incluindo reatores de tanque agitado (CSTR) sem e com reciclo de células, reatores em série, reatores sob vácuo e fermentadores do tipo torre com leveduras floculantes. Entretanto, existe um grande inconveniente, quando se utilizam biorreatores tubulares de leito fixo para produção de etanol, que é a formação de gás carbônico $\left(\mathrm{CO}_{2}\right)$ durante a fermentação. Este gás pode causar entupimento do gel bloqueando o escoamento do líquido e assim, 
tanto o líquido quanto o gás podem não escoar uniformemente através da coluna. De acordo com Pulitano (1990), este problema pode ser evitado usando-se separadores externos gás-liquido, os quais podem assegurar uma operação estável do biorreator.

Do ponto de vista de modelagem matemática, as cinéticas de fermentações alcoólicas têm sido geralmente descritas por meio de modelos não segregados e não estruturados nos quais, a população microbiana é considerada homogênea e não há variação da concentração dos componentes intracelulares (Astudillo e Alzate, 2011; Oliveira, 2000). Estes modelos são comumente representados por expressões dos tipos $\mu_{X}=f_{1}\left(C_{S}\right) g_{1}\left(C_{P}\right) h_{1}\left(C_{X}\right)$ e $\mu_{P}=f_{2}\left(C_{S}\right) g_{2}\left(C_{P}\right) h_{2}\left(C_{X}\right)$, onde $\mu_{x}$ e $\mu_{p}$ são as velocidades específicas de crescimento e formação de produto, $C_{S}$ é a concentração de substrato limitante, $C_{p}$ é a concentração de etanol e $C_{x}$ é a concentração de biomassa (Oliveira, 2000).

O efeito da concentração de substrato nas cinéticas de fermentação é geralmente descrito pela expressão de Monod, exceto para os casos de alta concentração de substrato ou limitações difusionais presentes em biomassas imobilizadas ou floculantes, quando os modelos de Andrews e Contois podem ser respectivamente utilizados. O efeito inibitório do etanol nas velocidades específicas de crescimento e formação de produto é reportado na literatura como sendo do tipo não competitivo e várias equações têm sido propostas para este efeito, incluindo expressões do tipo linear, exponencial, parabólico, hiperbólico e não linear generalizado (Luong, 1985). Para descrever o efeito inibitório devido ao excesso de biomassa tem sido freqüentemente utilizada uma equação do tipo lei de potência no termo $\left(1-C_{X} / C_{X, m}\right)$ (Lee, 1985). Para a descrição da cinética de consumo de substrato tem sido de modo geral utilizado o modelo de Herbert-Pirt, segundo o qual o substrato é consumido para crescimento e manutenção celular e para formação de produto (Oliveira, 2000):

Stremel (1994) desenvolveu um modelo matemático contendo 15 parâmetros para um biorreator tubular de leito fixo multiestágio com separadores externos gás-líquido, o qual foi utilizado para a produção contínua de etanol. Estes parâmetros incluem constantes cinéticas, fatores de conversão, coeficientes de difusão e dispersão. Devido à alta complexidade do modelo, apenas dois destes parâmetros foram otimizados sendo os demais determinados a partir de informações da literatura. Desta forma, o objetivo deste trabalho foi propor estratégias para a redução do modelo matemático do biorreator tornando-o menos complexo para uma futura otimização de parâmetros por técnicas clássicas de regressão não linear.

\section{MATERIAIS E MÉTODOS}

\subsection{Dados Experimentais}

Os dados experimentais utilizados neste trabalho foram obtidos por Pulitano (1994) operando um reator tubular de leito fixo mostrado esquematicamente na Figura 1. O reator consiste de quatro estágios e três separadores externos gás-líquido. No interior dos separadores há um mecanismo de controle automático do nível de líquido análogo a um sistema válvula-bóia. O reator e os separadores foram mantidos submersos em um banho termostático à temperatura de $30^{\circ} \mathrm{C}$. As células de levedura 
Saccharomyces cerevisiae foram imobilizadas em gel de pectina cítrica resultando em pellets de 3,0 a $3,5 \mathrm{~mm}$ de diâmetro. As entradas dos estágios foram bloqueadas com rolhas de borracha para evitar a saída dos pellets bem como a entrada de água no reator. As ligações entre os separadores e os estágios foram feitas por meio de mangueiras de silicone. Caldo de cana-de-açúcar foi alimentado continuamente na base do reator.

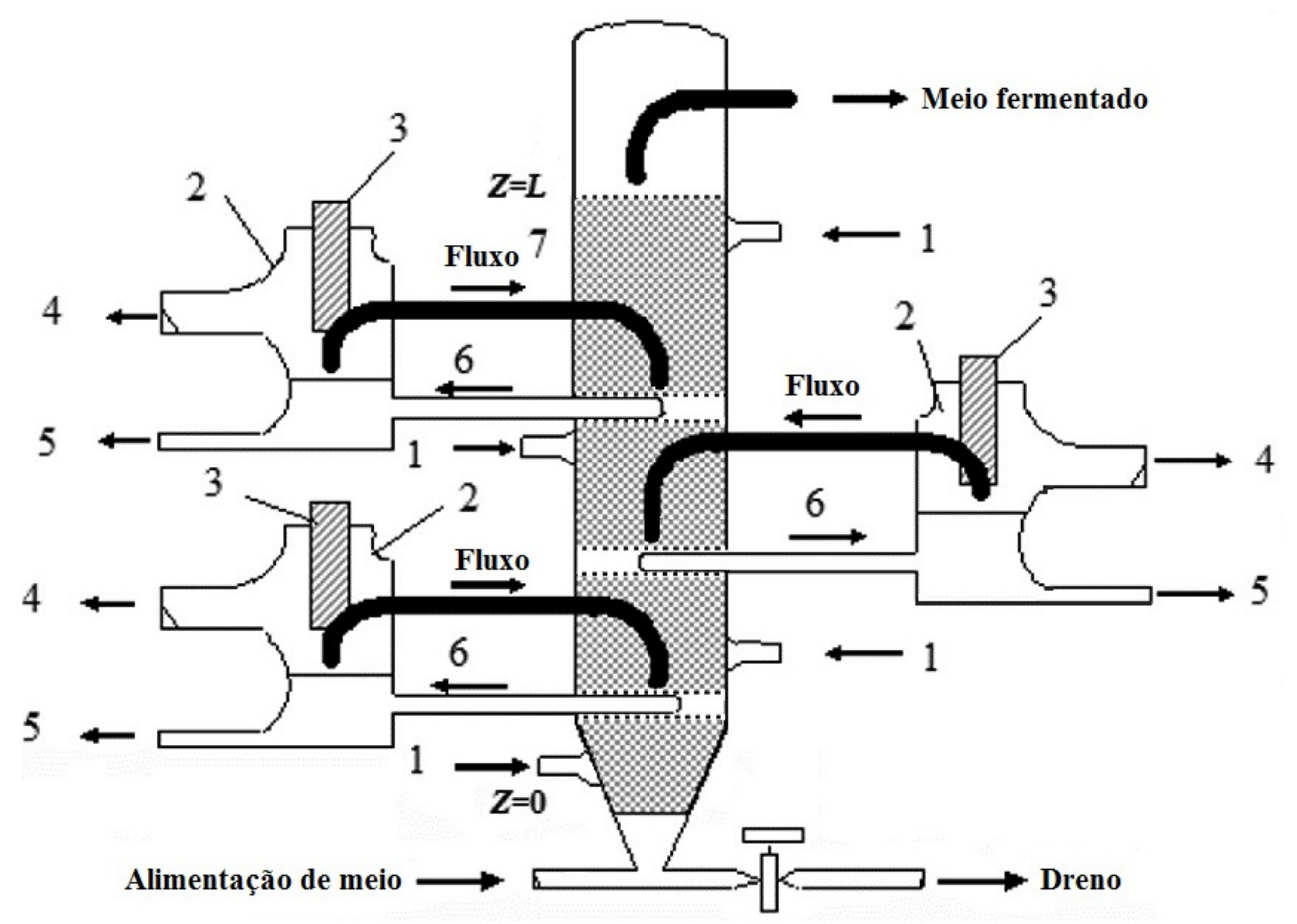

Figura 1 - Desenho esquemático do biorreator tubular de leito fixo multiestágio com separadores externos gáslíquido: 1-Entrada e saída de pellets; 2-Separador gás-líquido; 3-Sensor indutivo; 4-Saída de $\mathrm{CO}_{2}$; 5-Retirada de amostras; 6-Fluxo de líquido fermentado com $\mathrm{CO}_{2} ;$ 7-Fluxo de líquido sem $\mathrm{CO}_{2}$.

Os ensaios realizados por Pulitano (1990) permitiram a obtenção dos perfis axiais de concentração de substrato e etanol em regime transiente e estacionário para duas diferentes vazões e concentrações de substrato na alimentação.

\subsection{Modelo Matemático Reduzido}

Modelagem da fase sólida (partícula): Foi desenvolvido um modelo reduzido para a partícula devido a uma análise preliminar indicar que a resistência à transferência de massa interna à partícula é desprezível, pois o valor do fator de efetividade manteve-se muito próximo de um em todo o biorreator nas condições operacionais do processo, podendo-se, desta forma, desprezar os gradientes de concentração no modelo da partícula. Entretanto, um termo foi introduzido nas equações de balanço de massa das espécies difundentes para garantir que substrato entrasse e etanol saísse da partícula. 
Com estas premissas básicas, as equações de balanço de massa no interior da partícula em regime transiente são dadas pelas seguintes equações:

$$
\begin{aligned}
& \frac{d c_{X}}{d t}=\mu_{X} c_{X}-r_{d} ; \mu_{X}=\left(\frac{\mu_{\text {máx }} c_{S}}{K_{M} c_{X}+c_{S}+\frac{c_{S}^{2}}{K_{I}}}\right)\left(1-\frac{c_{X}}{c_{X}^{*}}\right) e^{-K_{P} c_{P}} ; r_{d}=k_{d} c_{X} \\
& \frac{d c_{S}}{d t}=k_{m_{S}} a\left(C_{S}-c_{S}\right)-\mu_{S} c_{X} ; \mu_{S}=\frac{\mu_{X}}{Y_{X / S}}+m \\
& \frac{d c_{P}}{d t}=\mu_{P} c_{X}-k_{m_{P}} a\left(c_{P}-C_{P}\right) ; \mu_{P}=Y_{P / S} \mu_{S}
\end{aligned}
$$

onde os termos convectivos $k_{m_{S}} a\left(C_{S}-c_{S}\right)$ e $k_{m_{P}} a\left(c_{P}-C_{P}\right)$ representam o fluxo de entrada de substrato e o fluxo de saída de etanol na partícula; $k_{m_{S}}$ e $k_{m_{P}}$ são os coeficientes de transferência de massa para o substrato e para o etanol, respectivamente; $\mu_{X}, \mu_{S}, \mu_{P}$, são as velocidades específicas de crescimento, consumo de substrato e formação de produto, respectivamente; $\mu_{\max }$ é a velocidade especifica máxima de crescimento; $r_{d}$ é a taxa de morte celular, $c_{X}$ é a concentração de microrganismos no interior da partícula; $C_{S}$ é a concentração de substrato na fase fluida; $c_{S}$ é a concentração de substrato no interior da partícula; $C_{P}$ é a concentração de produto (etanol) na fase fluida; $c_{P}$ é a concentração de produto no interior da partícula; $K_{M}$ é a constante de saturação do modelo de Monod; $k_{d}$ é a velocidade específica de morte celular; $m$ é a velocidade especifica de consumo de substrato para manutenção celular; $Y_{X / S}$ e $Y_{P / S}$ são os coeficientes aparentes de conversão de substrato em células e produto, respectivamente.

Modelagem da fase fluida: Para a modelagem da fase fluida aproximou-se o biorreator tubular por uma cascata de N-CSTRs em série. As seguintes equações representam os balanços de massa de substrato e etanol em cada estágio i da cascata de reatores:

$$
\begin{aligned}
& \frac{d C_{S_{i}}}{d t}=\frac{Q}{\varepsilon V_{i}}\left(C_{S_{i-1}}-C_{S_{i}}\right)-\eta\left(\hat{r}_{S}\right)_{\text {sup }}\left(\frac{1-\varepsilon}{\varepsilon}\right) ; i=1, \ldots . ., N \\
& \frac{d C_{P_{i}}}{d t}=\frac{Q}{\varepsilon V_{i}}\left(C_{P_{i-1}}-C_{P_{i}}\right)+Y_{P / S} \eta\left(\hat{r}_{S}\right)_{\text {sup }}\left(\frac{1-\varepsilon}{\varepsilon}\right)
\end{aligned}
$$


onde $Q$ é a vazão volumétrica de alimentação; $N$ é o número de reatores a ser estimado; $V_{i}$ é igual ao volume do reator tubular dividido por $N$; $\varepsilon$ é a porosidade; $\eta$ é o fator de efetividade; $\left(\hat{r}_{S}\right)_{\text {sup }}$ é a velocidade de consumo de substrato na superfície da partícula.

Para cada unidade da cascata de reatores em série foram obtidas 5 equações diferenciais ordinárias as quais representam os 3 balanços de massa na partícula (microrganismos, substrato e produto) e os dois balanços de massa na fase fluida (substrato e produto). No total tem-se $5 \times \mathrm{N}$ equações diferenciais ordinárias no tempo as quais foram integradas numericamente utilizando-se o método de Runge-Kutta-Gill de $4^{\mathrm{a}}$ ordem com passo de integração variável.

\subsection{Parâmetros utilizados na Simulação}

A Tabela 1 mostra os valores dos parâmetros e condições experimentais utilizadas na simulação do biorreator (Pulitano, 1990; Stremel, 1994). Nos itens onde há dois valores, o superior refere-se à operação com baixa concentração de substrato na alimentação e o inferior refere-se à operação com alta concentração.

Tabela 1 - Parâmetros e condições operacionais usados na simulação do biorreator

\begin{tabular}{|c|c|c|}
\hline \multicolumn{2}{|c|}{ PARÂMETROS } & CONDIÇÕES OPERACIONAIS \\
\hline $\begin{array}{l}\mu_{\text {max }}=0,45 \mathrm{~g} / \mathrm{g} \cdot \mathrm{h} \\
K_{M}=4,42 \mathrm{~g} / \mathrm{L} \\
K_{I}=216,0 \mathrm{~g} / \mathrm{L} \\
K_{p}=\left\{\begin{array}{l}0,03 \mathrm{~L} / \mathrm{g} \\
0,036 \mathrm{~L} / \mathrm{g}\end{array}\right. \\
{c_{X}^{*}}^{*}=80,0 \mathrm{~g} / \mathrm{L} \\
K_{d}=\left\{\begin{array}{l}0,0054 \mathrm{~g} / \mathrm{g} \cdot \mathrm{h} \\
0,0060 \mathrm{~g} / \mathrm{g} \cdot \mathrm{h}\end{array}\right. \\
m=\left\{\begin{array}{l}0,30 \mathrm{~g} / \mathrm{g} \cdot \mathrm{h} \\
0,36 \mathrm{~g} / \mathrm{g} \cdot \mathrm{h}\end{array}\right. \\
Y_{X S}=\left\{\begin{array}{l}0,022 \mathrm{~g} / \mathrm{g} \\
0,030 \mathrm{~g} / \mathrm{g}\end{array}\right.\end{array}$ & $\begin{array}{l}Y_{P S}=\left\{\begin{array}{l}0,48 \mathrm{~g} / \mathrm{g} \\
0,40 \mathrm{~g} / \mathrm{g}\end{array}\right. \\
d_{P}=\left\{\begin{array}{l}0,0356 \mathrm{dm} \\
0,0366 \mathrm{dm}\end{array}\right. \\
L=1,9 \mathrm{dm} \\
d_{R}=0,5 \mathrm{dm} \\
\varepsilon=\left\{\begin{array}{l}0,304 \\
0,256\end{array}\right. \\
\rho_{f}=1152,4 \mathrm{~g} / \mathrm{dm}^{3} \\
\mu_{f}=1980 \mathrm{~g} /(\mathrm{dm} \cdot \mathrm{h})\end{array}$ & $\begin{array}{l}Q=\left\{\begin{array}{l}40,0 \times 10^{-3} \mathrm{dm}^{3} / \mathrm{h} \\
49,4 \times 10^{-3} \mathrm{dm}^{3} / \mathrm{h}\end{array}\right. \\
C_{S_{0}}=\left\{\begin{array}{l}161,4 \mathrm{~g} / \mathrm{L} \\
312,5 \mathrm{~g} / \mathrm{L} \text { (alimentação) }\end{array}\right. \\
C_{X_{i}}=21,0 \mathrm{~g} / \mathrm{L} \text { (partícula) }\end{array}$ \\
\hline
\end{tabular}




\section{RESULTADOS E DISCUSSÃO}

Combinando-se as estratégias de redução do modelo da partícula e do modelo hidrodinâmico do biorreator, desenvolveu-se um modelo alternativo muito menos complexo, mas conservativo, o qual possibilitou a simulação do bioprocesso com um menor esforço computacional.

As Figuras 4 e 5 mostram o desempenho da cascata de CSTRs em série em função do número de reatores empregados $(\mathrm{N})$.
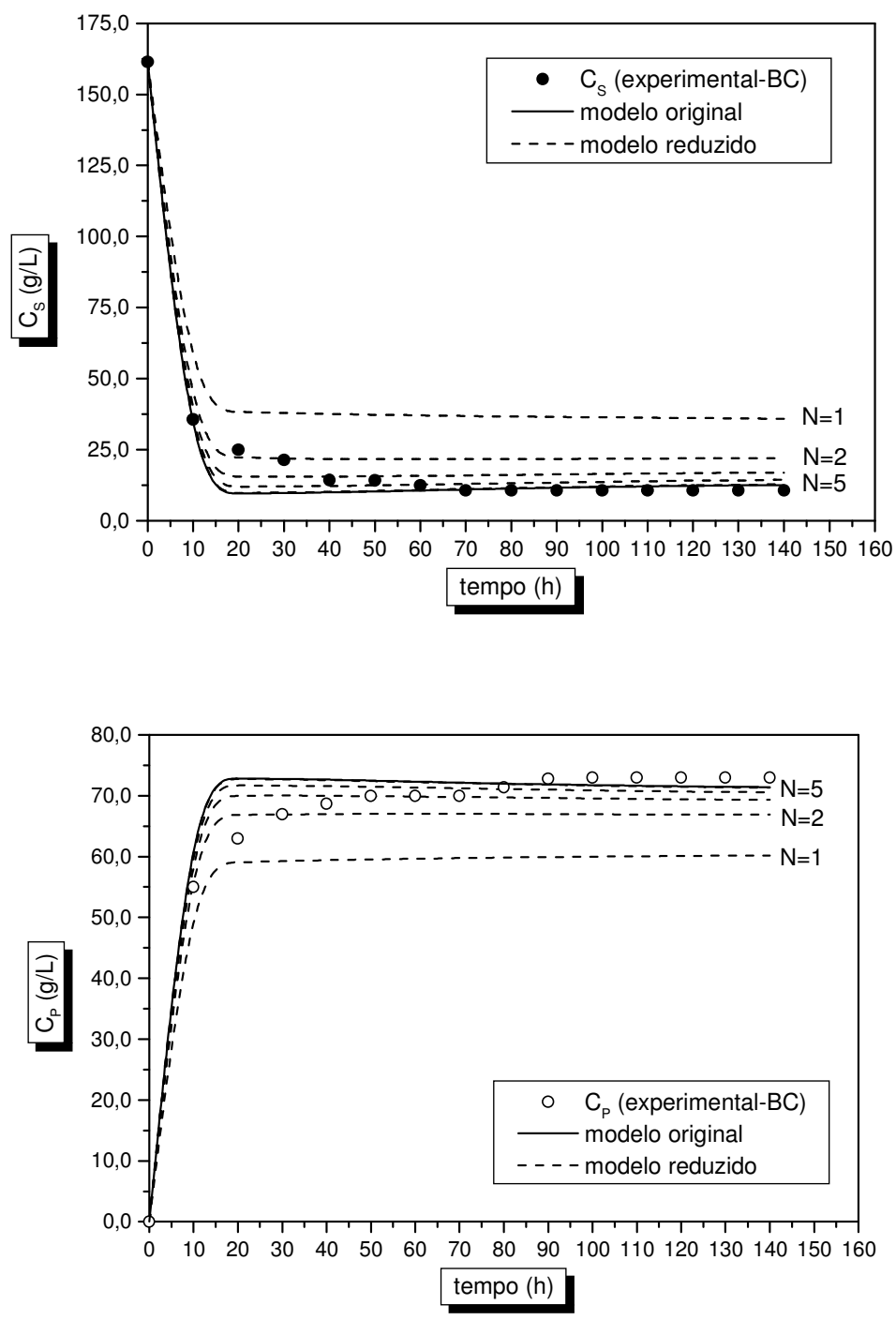

Figura 4- Perfis de concentração de substrato e etanol na saída da cascata de reatores no regime transiente para baixa concentração de substrato na alimentação 

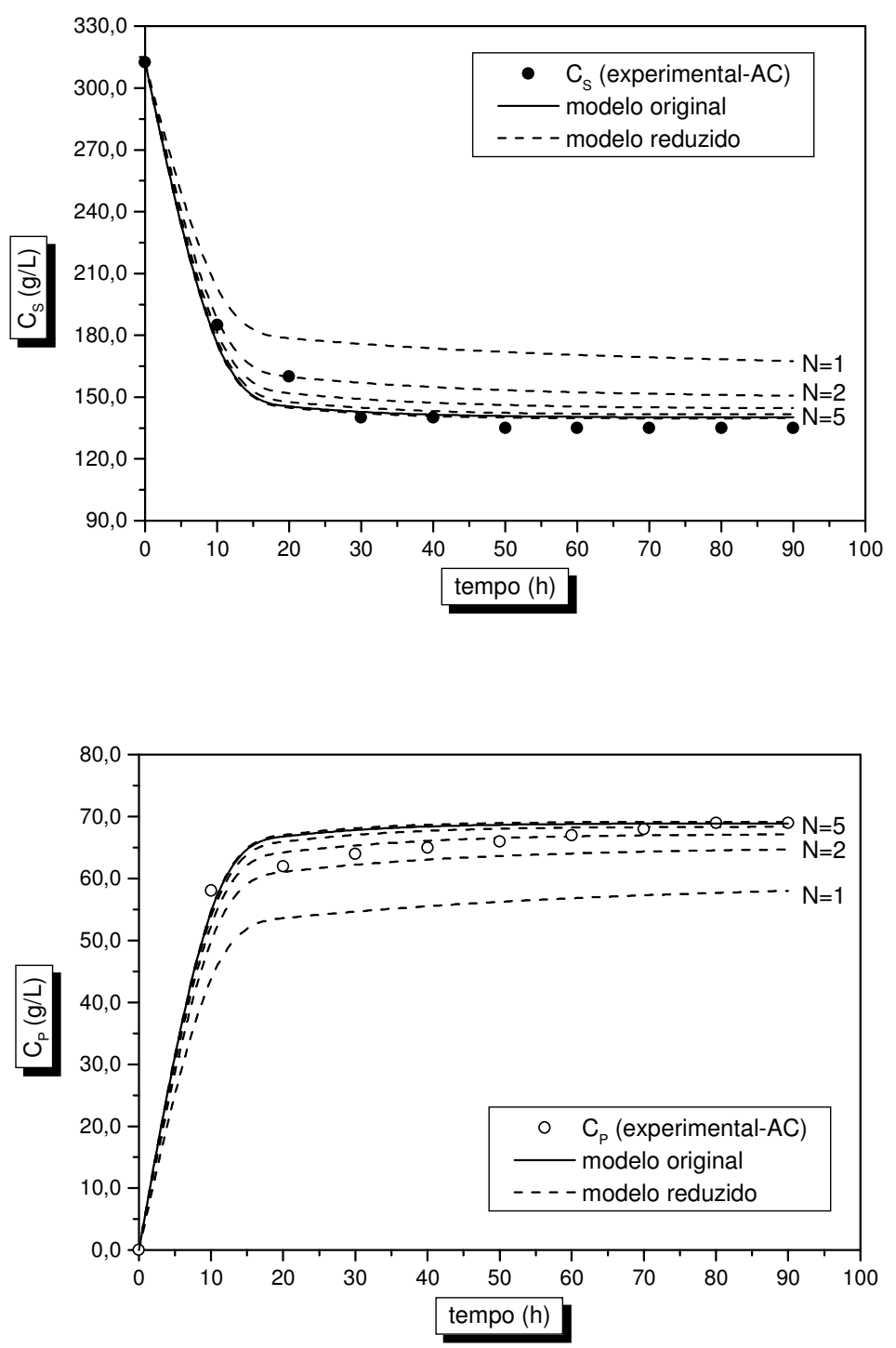

Figura 5- Perfis de concentração de substrato e etanol na saída da cascata de reatores no regime transiente para alta concentração de substrato na alimentação

Como pode ser observado, as previsões do modelo reduzido para $N=5$ coincidem com aquelas do modelo original tanto para baixa quanto para alta concentração de substrato na alimentação. Assim, o biorreator tubular pode ser aproximado por uma cascata de 5 reatores de mistura em série sem perda da capacidade preditiva do modelo reduzido, uma vez que as previsões deste modelo concordam bem com o comportamento dinâmico da concentração de substrato e de etanol medidas experimentalmente. 


\section{CONCLUSÕES}

Foram propostas estratégias de redução de modelos matemáticos aplicadas a um processo contínuo de fermentação alcoólica em biorreator tubular de leito fixo.

O modelo matemático original é bastante complexo e compreende equações diferenciais parciais tanto para a fase sólida quanto para a fase fluida, as quais surgem devido aos fenômenos de difusão-reação e dispersão axial serem considerados na modelagem do processo.

As estratégias de redução aplicadas consistiram em desprezar os gradientes de concentração no interior da partícula e aproximar o biorreator tubular de leito fixo por uma cascata de N-CSTRs em série de volume equivalente. Deste modo simplificou-se significativamente o modelo original, reduzindo-se o sistema inicial de EDPs a um sistema de EDOs.

Do ponto de vista de modelagem matemática, a redução de complexidade foi bastante significativa, pois se desenvolveu um modelo muito mais simples, mas conservativo, o qual pode ser usado para estudos de estimação de parâmetros, controle e otimização do bioprocesso com um menor esforço computacional.

\section{REFERÊNCIAS}

ASTUDILlO, I. C. P.; ALZATE, A. C. A. Importance of stability study of continuous systems for ethanol production. J. Biotechnol., v. 151, p. 43-55, 2011.

LUONG, J. H. T. Kinetics of ethanol inhibition in alcohol fermentation. Biotechnol. Bioeng., v. 27(3), p. $280-285,1985$.

LEE, J. M. Computer simulation in ethanol fermentation. In: Biotechnology Applications and Research, CHEREMISINOFF, P. N.; ONELLETTE, R. P. (eds.), Technomic Publishing Company Inc., p. 78-87, 1985.

OLIVEIRA, S. C. Modelagem matemática de um processo contínuo de fermentação alcoólica em reator tipo torre com reciclo de leveduras floculantes. São Paulo, 2000. Tese (Doutorado) - Escola Politécnica, USP.

PULITANO, V. M. S. E. Produção contínua de etanol em reator de alta produtividade utilizando levedura imobilizada em pectina cítrica. São Carlos, 1990. Dissertação (Mestrado) - Departamento de Engenharia Química, UFSCar.

STREMEL, D. S. Simulação dinâmica de um bioreator tipo torre com células imobilizadas para produção de etanol. São Carlos, 1994. Dissertação (Mestrado) - Departamento de Engenharia Química, UFSCar. 